\title{
Cervical spondylitic myeloradiculopathy due to chronic brucellosis in a Ugandan teenager
}

\author{
*Kyebambe $\mathrm{P}^{1}$, Kasyaba $\mathrm{R}^{1}$, Nkakyekorera $\mathrm{S}^{2}$ \\ 1. Internal Medicine department, Kabale Regional Referral Hospital \\ 2. Radiology department, Kabale Regional Referral Hospital \\ Keywords: Cervical spondylitis brucellosis myeloradiculopathy \\ African Health Sciences 2012; 12(3): 398 - 402 http://dx.doi.org/10.4314/ahs.v12i3.25
}

\section{Introduction}

Brucellosis is a worldwide zoonosis that causes much clinical morbidity as well as considerable loss of productivity in animal husbandry. It has been present for millennia and has managed to elude eradication even in most developed countries ${ }^{1}$ Transmission of brucellosis to humans occurs through the consumption of infected, un-pasteurized animal products, through ruptures in skin/mucous membranes in direct contact with infected animal parts and fluids (e.g placenta, urine, dung) and via inhalation of infected aerosolized particles.

Brucellosis is an occupational disease in herdsmen, abattoir/dairy workers, veterinarians, and microbiologic laboratory personnel .Consumption of unpasteurized dairyproducts e.g raw milk, yoghurt, and ice cream is the commonest means of transmission. Infection is also acquired through consumption of undercooked/under-roasted meats ${ }^{1}$. Human-to-human transmission through sexual fluids and breast milk has been documented ${ }^{2,3}$. Six species of brucella are known to cause human disease namely: B.melitensis(from goats,sheep,camels), B.abortus(from cows), B. suis(from pigs), B. canis(from dogs) and the recently discovered marine types: Brucella pinnipediae(from dolphins and whales) and B.cetaceae (from seals). The vast majority of cases worldwide are attributed to B. melitensis ${ }^{1}$. After entering the human body and being taken up by local tissue lymphocytes, brucellae are transferred through regional lymph nodes into the circulation and are

\author{
*Correspondence author: \\ Dr. Peterson Kyebambe \\ Department of Internal Medicine \\ Kabale Regional Hospital \\ P.O Box 7 \\ Kabale, Uganda \\ Telephone: +256 772448771 \\ Email: kyebsteve@yahoo.com
}

subsequently seeded throughout the body, with tropism for the reticuloendothelial system. The incubation period usually ranges from 2 to 4 weeks ${ }^{1}$. The disease is protean and complications can occur in any organ or body system namely: genitourinary, haematological, cutaneous and ocular ${ }^{1,4,5,6}$.

\section{Brucellosis in Africa}

Human brucellosis has been documented in Uganda and Africa for decades, however, the disease had never been considered to be a big public health problem, probably due to under-diagnosis ${ }^{5}$. Recently, there have been increased reports of this zoonosis on the African continent ${ }^{6-13}$.

\section{Objectives of reporting the case}

1. To raise awareness that brucellosis and its complications do exist in Africa.

2. To demonstrate that neurobrucellosis can resemble non-communicable diseases like Amyotrophic Lateral Sclerosis (ALS).

3. To share with readers one of our most spectacular clinical experiences and demonstrate to them that a diagnosis and cure of complicated

brucellosis is possible even in centres without sophisticated investigational facilities such as Computed Tomogram (CT)scan or Polymerase Chain Reaction (PCR).

\section{Case report}

In August 2006,an 18 year old girl was admitted to our unit with a 12 year history of recurrent multiple joint and muscle pains, fevers and sweats. There were radicular pains felt down both arms. She was so weak that she could not even button her dress and had eventually dropped out of school 8 years earliershe could not write or sit in class. After diagnosis, it was discovered that her family rears goats and the patient had been cleaning up animal excreta from 
the yard with her bare hands for years. The animals were not immunized against brucellosis.

There was no history of consuming raw dairy products or underroasted/undercooked meat. Examination revealed a sick-looking, wasted, pale and debilitated teenager. She had marked wasting and weakness of the upper limbs with attendant inability to use her hands normally (inset A). There was marked atrophy of the hypothenar and thenar eminences of both hands with resulting difficulty in abducting or adducting the fingers (inset A1), with hypotonia and diminished reflexes in both upper limbs. In the lower limbs there was reduced muscle power but increased tone and reflexes with an extensor plantar response. She had a sensory gait and sensory level at C8 (cervical dermatome 8). The mentation and cranial nerves were intact. Other body systems were unremarkable except for generalised bone/joint tenderness.

A clinical impression of chronic cervical cord and nerve root compression ${ }^{14,15}$ of uncertain cause was made. Cervical antero-posterior, lateral and oblique view $\mathrm{x}$-rays were taken revealing anterior ankylosis and narrowing between $\mathrm{C} 2$ and $\mathrm{C} 3$ plus reduced intervertebral spaces at C4/5,C5/6, C6/7 and $\mathrm{C} 7 / \mathrm{T} 1$ indicating multiple prolapsed intervertebral discs and myelopathy at those levels (inset C). The foramina and chest $\mathrm{x}$-ray were normal. A clinical-radiological diagnosis of cervical spondylitic myelo-radiculopathy was made ${ }^{14,15}$. Advanced tests such as CT scan could not be done as they are not available in our locality (nearest CT is $400 \mathrm{~km}$ away).

The $\mathrm{Hb}=10.8$ grams per dl, Erythrocyte Sedimentation Rate $($ ESR $)=94 \mathrm{~mm}$ after one hour (normal $=0-20 \mathrm{~mm}$, Westergren). The total leukocyte count was 3600 cells per $\mathrm{ml}$ total (normal is 4000 11,000 ) with a lymphocytosis of $63.3 \%$ (normal is 20-40\%). The brucella Serum Agglutination Test (SAT) done (Cypress Diagnostics, Belgium) was significantly reactive with a titre of 1: 320 after 72 hours of incubation (significant titre $=1: 160$ or more $)^{1,4}$. There were no facilities for brucella culture or PCR. We could not do a lumbar puncture to obtain cerebrospinal fluid (CSF) for fear of aggravating the cord compression yet we do not have neurosurgical capacity. The serological tests for HIV (Determine-Abbott, Japan and StatpakChembio, USA) were both negative. The serological tests for tuberculosis ('Hexagon

TB'-Human, Germany), syphilis (RPR, TPHAAbbott, Japan) and Systemic Lupus (Anti-Nuclear Antibody-Human,Germany ) were all negative.

Figure 1: cervical spondylitic myeloradiculopathy due to chronic brucellosis in a teenage Ugandan female

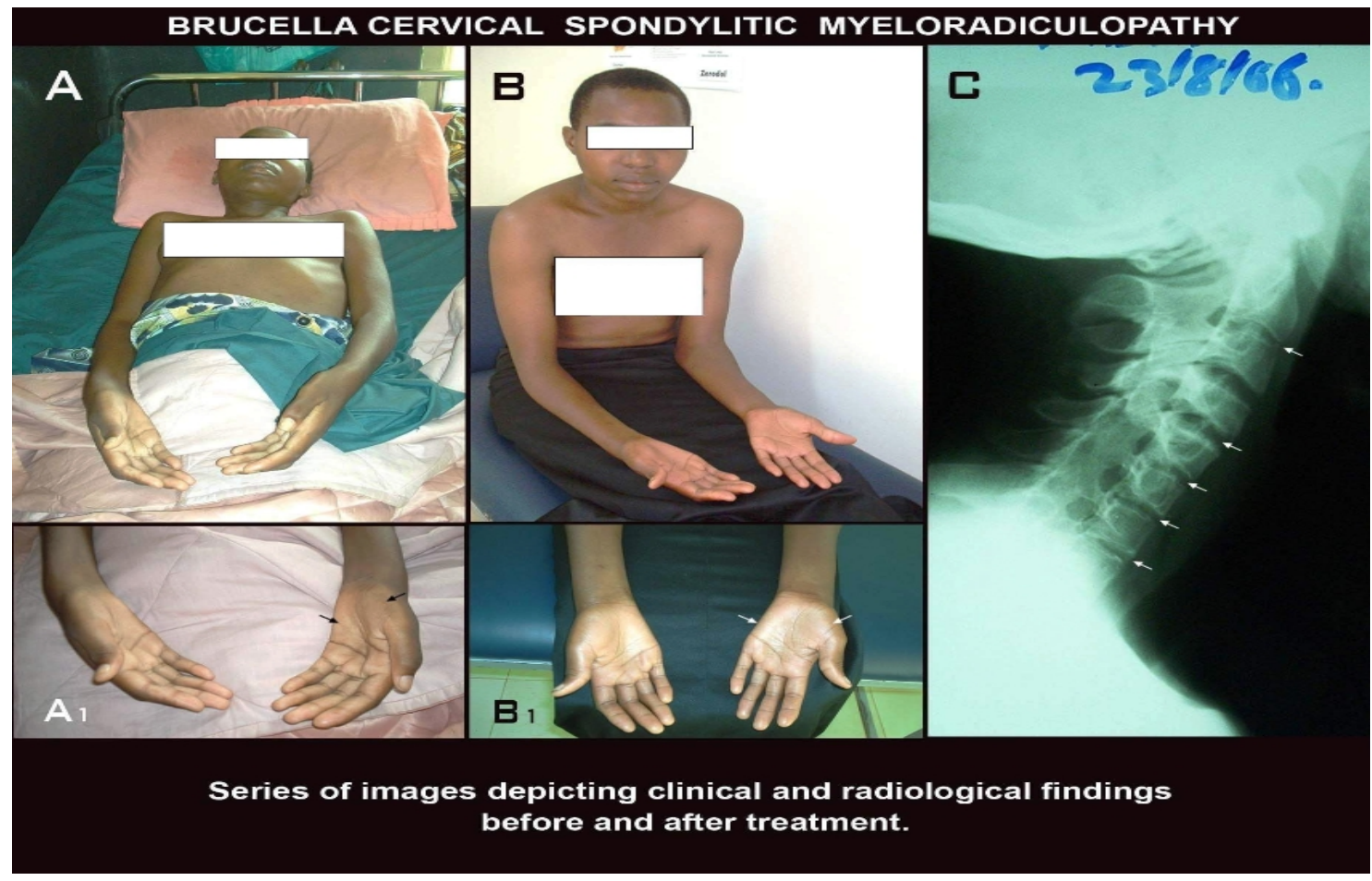

$649 \times 589 \mathrm{~mm}(72 \times 72 \mathrm{DPI})$ 


\section{Results}

Treatment was started with 3 drugs: intramuscular (IM) streptomycin 1gram OD for 2 weeks together with oral doxycycline $200 \mathrm{mg}$ OD and oral ofloxacin $200 \mathrm{mg}$ BD for a total of 20 weeks. Streptomycin was stopped after the first 2 weeks ${ }^{1,4,16}$ and replaced by oral rifampicin $600 \mathrm{mg}$ OD so as to maintain a minimum of three drugs $s^{1,16,17}$. Therapy was continued until irrevocable evidence of healing had been noted and the serology had turned negativethis occurred after 20 weeks (inset B). The general body, thenar and hypothenar eminences regained normal size and function (inset B1).

We have followed this patient post-treatment for over 36 months now and she has continued to do well. The family has been counselled about prevention of brucellosis.

\section{Discussion}

\section{Manifestations of neurobrucellosis}

Neurobrucellosis occurs in 3-7\% of all patients with brucellosis ${ }^{1,3,4,4,21}$.The commonest manifestations are meningitis and meningoencephalitis followed by myelitis, craniopathies, peripheral neuropathy, radiculopathy, cerebellar ataxia, meningovascular complications (including mycotic aneurysms, ischaemic strokes and subarachnoid hemorrhage). Rarer manifestations include isolated intracranial hypertension, Guillain-Barre syndrome, brain abscesses, diabetes insipidus, cerebral venous thrombosis, subdural hemorrhage and psychiatric syndromes. CSF examination shows elevated protein, low glucose and a mononuclear pleocytosis. The SAT is frequently positive but brucella culture in CSF is only positive in $20 \%$ of cases $^{21}$. The case we are documenting was unusual in that symptoms lasted over 12 years without the diagnosis being made and the clinical picture resembled an incurable disease like ALS. In the medical literature, there are only two African case reports of neurobrucellosis, one of which was also from our unit ${ }^{22,23}$. Despite brucellosis being an endemic infection in Africa, many health care providers are not conversant with it. This patient had visited several health units in the locality over the years, but nobody ever suspected that brucellosis could be the cause of her persistent illness.

We aim to alert health personnel in Africa and other areas, about the protean nature of brucellosis and its ability to cause chronic disease including crippling neurological manifestations.
Tuberculosis (TB) is an important differential in spondylitic brucellosis and it may be difficult to differentiate the two clinically in a resourcelimited setting. However, evidence from some workers (including ourselves) indicates that this can be possible on plain radiographs - TB tending to cause early erosions of the anterior aspect of the vertebrae with consequent bone destruction, vertebral collapse, wedging and gibbus formation unlike brucellosis which usually does not behave likewise et,25,26,27. $^{2}$

\section{Treatment of neurobrucellosis (including myelopathy)}

It is recommended to use at least 3 classes of drugs at any one time toprevent development of resistance and relapse $e^{1,16,17,20}$. In neurobrucellosis at least 2 of the drugs should be able to cross the blood-brain barrier. Drugs that can do so include third generation cephalosporins (e.g ceftriaxone,cefixime), tetracyclines (usuallydoxycycline),rifamycins (usually rifampicin) and cotrimoxazole ${ }^{1,16}$.Caution has to be taken to exclude tuberculosis before using rifamycins because of the danger of development of resistance to rifampicin by TB bacteria if its used without other anti-tuberculous drugs in a patient with active TB ${ }^{1}$. Our therapy was successful because it continued until all signs and laboratory evidence (SAT) had cleared. Using serology alone as an indicator of successful treatment may be misleading as it can remain positive for months even after the infection has been eliminated from the patient. We followed the experiences of other clinicians in the medical literature whose subjects cured/improved after treatment for longer than the traditionally recommended 6-8 weeks ${ }^{17,18,19}$. At 6 weeks of therapy our case had made only a little improvement. Our findings agree with those of other authors whose patients cured/ improved after these durations: Solera(45-535days), Bodur (5 months) and Gul (2-15 months) ${ }^{[17,18,19]}$.

Serious side/toxic effects of the drugs we used include: allergy, hepatitis(rifampicin), vomiting,benign intracranial hypertension (doxycycline),psychosis (ofloxacin).Our patient was monitored monthly clinically, no significant side/toxic effect was reported or noted.

\section{Learning points}

1. Brucellosis is a common and often missed diagnosis in Africa.

2. Diagnosis and cure of complicated brucellosis is possible even in units without sophisticated facilities. 
3. In complicated brucellosis, treatment should continue until all the clinical symptoms and signs completely resolve.

4. The people, the governments and many health workers are not aware of the presence of brucellosis and its very diverse and often deleterious manifestations. Awareness and action need to be promoted to diagnose, manage and prevent this curable but debilitating/lethal zoonosis.

\section{References}

1. Pappas G, Akritidis N, Bosilkovski M, Tsianos E. Brucellosis. N Engl J Med.2005 Sep 8; 353(10):1071- 2; author reply1071-2.

2. Wyatt HV.Brucella melitensis can be transmitted sexually. Lancet 1996 Aug 31;348(9027):615.

3. Gür A, Geyik MF, Dikici B, Nas K, Cevik R, Sarac J,et al.Complications of brucellosis in different age groups: a study of 283 cases in southeastern Anatolia of Turkey. Yonsei Med J. 2003 Feb;44(1):33-44.

4. Young EJ. Brucella species. In Mandell, Douglas and Bennett's Principles and Practices of Infectious Diseases.7th 4th ed. Churchill Livingstone. 2009: 2921- 2925. 2386-2391.

5. Cox PS. Brucellosis-a survey in South Karamoja. East Afr Med J.1966; 43(2):43-50.

6. Nas K, Bükte Y, Ustün C, Cevik R, Geyik MF, Batmaz I. A case of brucellar spondylodiscitis involving the cervical spine. J Back Musculoskelet Rehabil. 2009;22(2):121-3.

7. Ndyabahinduka DG,Chu IH.Brucellosis in Uganda. Int JZoonoses. 1984Jun;11(1):59-64.

8. Schelling E,Diguimbaye C,Daoud S,Nicolet J,ZinsstagJ. Seroprevalences of zoonotic diseases in nomads and their livestock in ChariBarguimi,Chad. Med Trop (Mars).2004;64(5):4747.

9. Aggad H, Boukraa L. Prevalence of bovine and human brucellosis in western Algeria: comparison of screening tests. East Mediterr Health J. 2006; 12(1-2):119-28.

10. Kassahun J,Yimer E, Gevid A et al.Seroprevalence of brucellosis in occupationally exposed people in Addis Ababa,Ethiopia. Ethiop Med J. 2006; 44(3):245-52.

11. Meky FA,Hassan EA,Abd Elhafez AM,Aboul Fetouhl AM,El- Ghazali SM. Epidemilogy and risk factors of brucellosis in Alexandria governorate. East Mediterr Health J. 2007; 13(3):677-85.
12. Kang'ethe EK, Ekuttan CE, Kimani VN, Kiragu MW. Investigations Into the prevalence of bovine brucellosis and the risk factors that predispose humans to infection among urban dairy and non-dairy farming households in Dagoretti Division, Nairobi, Kenya. East Afr Med J.2007;84(11 Suppl):s96-100.

13. Kunda J, Fitzpatrick J, Kazwala et al. Healthseeking behaviour of human brucellosis cases in rural Tanzania. BMC Public Health.2007;7:315.

14. Clarke CRA. Neurological disease: Plexus and nerve root lesions. In Kumar P,Clark M, eds. Clinical Medicine.5th ed. International Edition. London, W.B Saunders. 2002:1217-1219.

15. Harrop JS,Hanna A,Silva MT,Sharan A. Neurological manifestations of cervical spondylosis: an overview of signs, symptoms and pathophysiology.c86. Neurosurgery.2007; 60(1 Suppl 1):S14-20.

16. World Health Organisation(WHO), World Organisation for Animal Health(oie),Food and Agriculture organisation of the United Nations(FAO). Brucellosis in humans and animals.2006edition.Geneva,Switzerland.WHO/ CDS/EPR/2006.7

17.Solera J, Lozano E, Martinez-Alfaro E, Espinosa A, Castillejos ML, Abad L. Brucellar spondylitis: review of 35 cases and literature survey. Clin Infect Dis.1999;29(6):1440-9.

18.Bodur H, Erbay A, Akinci E, Colpan A, Cevik MA, Balaban N. Neurobrucellosis in an endemic area of brucellosis. Scand J Infect Dis. 2003;35(2):94-7.

19. Gul HC, Erdem H, Bek S. Overview of neurobrucellosis: a pooled analysis of 187 cases. Int J Infect Dis. 2009; 13(6):e339-43.

20. Geyik MF, Gür A, Nas K, Cevik R, Saraç J, Dikici B, Ayaz C. Musculoskeletal involvement of brucellosis in different age groups: a study of 195 cases. Swiss Med Wkly. 2002; 132(7-8):98105.

21. Haji-Abdolbagi M,Rasooli-Nejad M, Jafari S, Hasibi M, Soudbaksh A. Clinical and laboratory findings in neurobrucellosis: review of 31 cases. Arch Iran Med.2008;11(1):21-5.

22.Kyebambe PS. Acute brucella meningomyeloencephalo-spondylosis in a teenage male. Afr Health Sci. 2005;5(1):69-72.

23. Hesseling AC, Marais BT, Cotton MF.A child with neurobrucellosis. Ann Trop Paediatr.2003;23(2):145-8. 
24.Turunc T, Demiroglu YZ, Uncu H, Colakoglu S, Arslan H. A comparative analysis of tuberculous, brucellar and Pyogenic spontaneous spondylodiscitis patients. Infect.2007;55(2):158-63.

25.Shanley DJ. Tuberculosis of the spine: imaging features. AJR Am J Roentgenol.1995 Mar;164(3):659-64.

26.Namiduru M, Karaoglan I, Gursov S, Bayazit N, Sirikci A. Brucellosis of the spine:evaluation of the clinical, laboratory and radiological findings of 14 patients. Rheumatol Int.2004;24(3):125-9. 27.Fitzgerald DW,Sterling TR,Haas DW. Mycobacterium tuberculosis, In Mandell, Douglas and Bennett's principles of Infectious Diseases. $7^{\text {th }}$ ed. Churchill Livingstone.2009:31293163. 